\title{
CONTRIBUTIONS A L'ÉTUdE DU CALCIUM DU LAIT (1)
}

\author{
par \\ RADU VLADESCO
}

Le calcium se trouve dans le lait sous forme de diverses combinaisons, parmi lesquelles les phosphates bi- et tricalciques et le caséinate de calcium sont unanimement admises. Mais en dehors de celles-ci, on trouve encore, d'après certains auteurs, du citrate de calcium et même du chlorure de calcium.

Quant aux proportions dans lesquelles ces combinaisons existent dans le lait, voici quelques données numériques :

D'après SOLDNER

Phosphate bicalcique .... 0,671

Phosphate tricalcique ... 0,806

Citrate de calcium....... 2,133

Chaux de la caséine ..... 0,465
D'après Van SLYKE et Bosworth

Phosphate bicalcique .... 1,750

Chlorure de calcium ..... 1,190

Chaux de la caséine ..... 0,540

D'après Ch. Porcher

$\begin{array}{lll}\text { Phosphate bi-et tricalcique } \ldots \ldots \ldots \ldots \ldots & 1,13 \\ \text { Citrate de calcium } \ldots \ldots \ldots \ldots \ldots \ldots \ldots \ldots & 1,80 \\ \text { Chaux de la caséine } \ldots \ldots \ldots \ldots \ldots \ldots \ldots \ldots & 0,60\end{array}$

Comme on le voit, il n'y a pas d'accord quant à la répartition du calcium entre ses divers sels.

De telles tentatives de répartition des cations entre les divers anions existant dans le lait, sont d'ailleurs considérées comme illusoires par certains auteurs comme Grimmer et W. M. Clark.

Cela à juste titre, car la plupart de ces combinaisons sont plus ou moins ionisées.

$$
\text { *** }
$$

Considérant l'état physique dans lequel ces combinaisons se trouvent dans le lait, elles peuvent être classées en deux catégories :

$1^{\circ}$ Des composés en solution cristalloïde.

$2^{\circ}$ Des composés en solution colloïde.

Dans la première catégorie rentrent le citrate de calcium et le phosphate de calcium au moins en partie et le chlorure de calcium s'il en existe. Dans la seconde catégorie, rentrent le phosphate tricalcique et le caséinate de calcium. Ces deux derniers composés sont des colloïdes micellaires différents entre eux par le fait que le premier est irréversible tandis que le second est réversible.

Dans le lait, le phosphate tricalcique est protégé par le caséinate

(1) Bulletin de l'Académie de Médecine de Roumanie, $3^{\mathrm{e}}$ annóe, t. VI, n 6, 7 décembre 1938, p. 822-827. 
de calcium et le complexe formé par eux est, à son tour, protégé par les colloïdes moléculaires, c'est-à-dire par l'albumine et par la globuline. En ce qui concerne la nature du complexe : caséinate de calcium + phosphate tricalcique, il est fort probable que ses constituants ne sont pas combinés chimiquement.

$$
* * *
$$

La quantité de calcium fixée par la caséine à l'état de caséinate est dépendante de la réaction du milieu aqueux dans lequel le complexe "caséinate + phosphate " est dissous.

A ce point de vue, les résultats des recherches faites par PALMER et par Ch. PORCHer sont très démonstratifs.

Voici les teneurs en calcium du caséinate sous différentes concentrations en ions hydrogène :

$\begin{array}{ll}p H & \mathrm{Ca} \% \\ \mathbf{5 , 8 0} & 0,593 \\ \mathbf{6 , 2 5} & 1,000 \\ \mathbf{6 , 4 5} & 1,071 \text { (neutralité au tournesol) } \\ \mathbf{7 , 0 0} & 1,400 \\ \mathbf{7 , 4 5} & 1,600 \\ 8,15 & 1,643 \text { (neutralité à la phénolphtaléine) } \\ 8,80 & 2,000 \\ \mathbf{9 , 3 5} & 2,380 \\ \mathbf{9 , 5 0} & \mathbf{2 , 6 1 0} \\ \mathbf{9 , 7 5} & \mathbf{2 , 9 7 0}\end{array}$

Le fait n'est pas du tout surprenant lorsqu'on pense que la easéine est un ampholyte d'un poids moléculaire d'environ 12.000 possédant 7 groupements aminés et 19 groupements carboxyliques libres.

La réaction du lait variant d'une façon continue, comme conséquence de la fermentation lactique du lactose, la teneur en calcium de la caséine, elle aussi, doit varier de la même manière.

Dans ces conditions, on comprend combien délicat est le dosage de la fraction du calcium provenant du caséinate de calcium.

$$
* * *
$$

D'après Ch. Porcher, le caséinate naturel contient environ $2 \%$ de calcium (évalué en $\mathrm{CaO}$ ) de sorte que dans 1 litre de lait, dont la teneur en ealcium total est de 1,75 (exprimé toujours en $\mathrm{CaO}$ ), 0,60 représente le calcium du caséinate (la teneur moyenne du lait en caséine est de 30 grammes par litre) et le reste, c'est-à-dire $1,75-0,60$, représente ce qu'on appelle ordinairement le calcium minéral. Ces chiffres se rapportent au lait de vache. 
Le lait de femme est beaucoup moins riche en calcium (il contient environ 0 gr. 38 de $\mathrm{CaO}$ par litre). En admettant que la easéine du lait de femme ait la même teneur en calcium que la caséine du lait de vache, il ressort que dans un litre de lait de femme il y a $0 \mathrm{gr} .160$ de $\mathrm{CaO}$ sous forme de caséinate (le lait de femme contient environ 8 grammes de easéine par litre) et 0 gr. 22 de $\mathrm{CaO}$ sous forme de sels (le calcium minéral).

$$
\text { ** * }
$$

Le dosage du calcium combiné à la caséine, quoique un peu plus compliqué, peut être fait après la séparation de la caséine. Nousmême, avons donné en 1935 une technique permettant cette opération [1].

Le dosage du calcium total, bien que plus facile, n'est fait que très rarement dans les analyses courantes. Pourtant il ne devrait être jamais négligé, étant donné l'importance de cet élément pour l'organisme animal et pour l'homme en particulier. Cela d'autant plus que les cas de carence alimentaire en calcium sont plus fréquents qu'on ne le pensait jusqu'à ce jour.

Il me semble que ce fait peut être attribué toujours à la méthode de dosage. En effet, le dosage du calcium, tel qu'on l'a fait jusqu'à présent, implique quelques opérations préliminaires d'une durée assez longue et qui nécessitent un outillage dont on ne dispose pas partout.

Il s'agit de l'évaporation du lait et ensuite de son incinération, opérations qui demandent au moins 4-5 heures.

$$
\text { ** }
$$

La méthode que nous avons préconisée [2] pour la défécation des liquides biologiques, c'est-à-dire la précipitation des protides à l'aide du sulfate de cuivre et du ferrocyanure de potassium, simplifie de beaucoup le dosage du ealcium. Cela pour la raison que cette méthode rend inutiles les opérations indiquées auparavant : la dessic cation et l'incinération de l'échantillon de lait à analyser.

L'expérience nous a prouvé en effet que la plus grande partie du calcium total passe dans le liquide séparé par filtration du liquide déféqué. Il est vrai qu'il reste dans le précipité une partie du calcium, mais elle peut être enlevée par le lavage du précipité avec certaines solutions salines.

En nous inspirant d'un travail extrêmement intéressant à ce point de vue [3], nous avons essayé plusieurs solutions salines et nous nous sommes arrêté à l'azote d'ammonium qui nous a donné des résultats tout à fait satisfaisants. La solution de ce corps, à raison de $10 \%$ et chauffée jusqu'à l'ébulition, enlève en effet tout le calcium retenu dans le précipité. 
Il est vraisemblable qu'il se passe dans ce cas un phénomène analogue à celui qui s'accomplit dans la terre arable, avec les silicates d'aluminium et de calcium (les zéolites) lorsqu'ils arrivent en contact des solutions des sels alcalins (le calcium passe en solution, tandis que le métal alcalin est insolubilisé).

$$
* *
$$

La technique du dosage est la suivante : dans un flacon jaugé de $100 \mathrm{~cm}^{3}$, on prend $10 \mathrm{~cm}^{3}$ de lait, on ajoute d'abord environ $70 \mathrm{~cm}^{3}$ d'eau distillée et ensuite $3 \mathrm{~cm}^{3}$ de solution saturée de sulfate de cuivre, et $2 \mathrm{~cm}^{3}$ de solution saturée de ferrocyanure de potassium. On complète le volume à $100 \mathrm{~cm}^{3}$ avec de l'eau distillée jusqu'au trait et on agite vigoureusement le mélange, en renversant plusieurs fois le flacon.

Après 10 minutes, on filtre, à travers un papier à filtrer, sur du zinc en poudre, afin d'enlever le cuivre qui pourrait nuire dans les opérations suivantes. Du liquide clair, séparé par filtration, on prend $25 \mathrm{~cm}^{3}$, on lui ajoute $2 \mathrm{~cm}^{3}$ d'acétate de sodium en solution saturée et $5 \mathrm{~cm}^{3}$ de solution d'oxalate d'ammonium à $5 \%$.

Le précipité d'oxalate de calcium, séparé après quelques heures de repos, est lavé trois fois avec la solution ammoniacale à $2 \%$ afin d'enlever toute trace d'oxalate d'ammonium. On dissout le précipité à l'aide de l'acide sulfurique étendu et on chauffe la solution obtenue jusqu'à l'ébulition. L'aeide oxalique mis en liberté est titré avec la solution de permanganate de potassium eentième normal, dont chaque $\mathrm{cm}^{3}$ correspond à $0 \mathrm{mgr}$. $2 \mathrm{Ca}$ ou à $0 \mathrm{mgr} .28 \mathrm{CaO}$.

A titre d'exemple, nous donnons les résultats de quelques expériences comparatives.

1. Lait de vache déféqué comme ci-dessus. Précipité lavé avec la solution bouillante d'azotate d'ammonium $10 \%$ :

Chaux par litre : 1 gr. 708.

Mêmè lait déféqué de la même manière. Sans lavage du précipité :

Chaux par litre:-1 gr. 428.

Même lait desséché et incinéré :

Chaux par litre : 1 gr. 680.

2. Lait de vache. Défécation cuivro-ferrocyanique. Précipité lavé avec la solution bouillante d'azotate d'ammonium à $10 \%$ :

Chaux par litre: 1 gr. 870.

Même lait. Même défécation. Sans lavage du précipité :

Chaux par litre: 1 gr. 568. 
Même lait desséché et incinéré :

Chaux par litre: 1 gr. 859.

(Laboratoire de chimie biologique Faculté de Médecine vétérinaire de Bucarest.)

\section{BIBLIOGRAPHIE}

[1] R. Vladesco. Une technique simple permettant l'analyse du lait dans un échantillon très réduit de ce liquide. Le Lait, n ${ }^{\circ} 144$, avril 1935, p. 363.

[2] R. Vladesco. Un défécant nouveau et ses applications en biochimie. Compte rendu Société Biologique, t. CXIX, 1935.

[3] R. Toporesco. Sur l'entraînement de la chaux et de la magnésie par le précipité d'oxyde ferrique. Compte rendu, Académie des Sciences, t. CLXX, 1920.

\section{LAITERIE EXPÉRIMENTALE DU GOUVERNEMENT HILLEROD (DANEMARK)}

16 RAPPORT

présenté par

le Professeur N. KJ ARGAARD-JENSEN

(Fin.)

\section{Résultats d'expériences}

\section{a) Influence de la stassanisation sur la flore bactérienne du lait.}

1. Expériences concernant la destruction des bacilles de la tuberculose.

Ainsi que les recherches précédentes, celles-ci ont été faites par le regretté Professeur Oluf BANG, dont le rapport prouve que l'appareil Stassano fonctionne d'une manière sûre en ce qui concerne la destruction des bacilles de la tuberculose aux températures auxquelles les expériences ont été faites, soit 73 et $75^{\circ} \mathrm{C}$., durée de chauffage 1 seconde 4.

Rapport du Laboratoire de Pathologie spéciale de l'Institut royal

Vétérinaire et Agronomique de Copenhague :

Expériences faites avec le stassaniseur du nouveau modèle.

Le 6 juillet 1936. - 50 litres de lait provenant de 2 vaches atteintes de tuberculose du pis furent mélangés à la Laiterie expérimentale du Gouvernement danois d'Hillerød, avec environ 550 litres de lait écrémé pasteurisé. Un échantillon de ce mélange fut prélevé. Du lait restant, une partie fut chauffée dans le stassaniseur à $75^{\circ}$, une partie à $73^{\circ}$. Des échantillons furent prélevés du lait chauffé à l'une et l'autre de ces températures. 